\title{
Morphology of thin films of lamellar diblock copolymers
}

\author{
Y. S. Kim, K. Y. Suh, and Hong H. Lee \\ Department of Chemical Engineering and Nanoelectronics Institute, Seoul National University, Seoul 151-742, Korea
}

(Received 1 May 1997)

\begin{abstract}
The morphology of unconfined thin films is determined for lamellar diblock copolymers. A criterion is developed for the conditions for island formation or flat surface. Also derived is the critical thickness of the film below which the morphology goes through a change from the flat surface to island structure. The period of underlying lamellar structure when islands form is determined. [S1063-651X(97)07610-1]
\end{abstract}

PACS number(s): 61.41.+e, 68.15.+e

It is well known that the morphology of thin films of diblock copolymers is different from that of bulk copolymer. In thin films, surface and strain effects due to lattice mismatch come into play. Various morphologies that result when a thin film of lamellar diblock copolymer is confined between two hard parallel surfaces have been studied [1-3]. When the film surfaces are not confined, however, different morphologies result. For thin films on a substrate with a free exposed upper surface, islands (or holes) form on the free surface due to stress [4-8]. The formation of islands (or holes) requires the interface to deform and stretch (increase area), as shown in Fig. 1, and this deformation increases the surface energy. On the other hand, the formation leads to relaxation of strain such that the total energy decreases in the process of island formation. It is well known that the height of the island is equal to the equilibrium lamellar period $L_{0}$ $[4,5,7,9,10]$. It is not known, however, what the period is for the underlying lamellar structure. While such a morphology has been observed for thin films, a flat free surface results when the film is relatively thick [11].

In this paper a criterion is derived for the conditions for island formation or flat surface. In the process, the question regarding the period of underlying lamellar structure is resolved.

We define the lattice mismatch $f$ as follows:

$$
f=\left(L_{0}-L\right) / L_{0},
$$

where $L_{0}$ is the equilibrium period of the diblock copolymer and $L$ is the period adjusted to film thickness. Then the strain energy in one dimension, $E_{\mathrm{st}}$, is given by

$$
E_{\mathrm{st}}=\frac{1}{2} G f^{2} t A,
$$

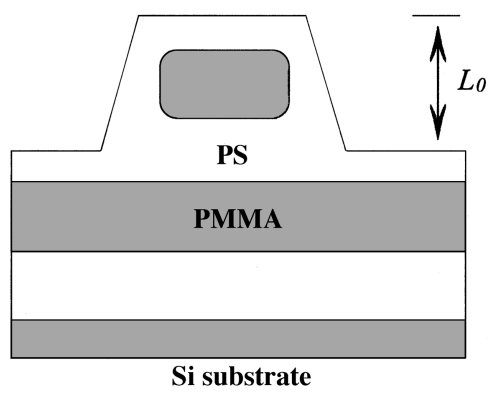

FIG. 1. Island formation in diblock copolymer film at equilibrium.

where $G$ is Young's modulus, $t$ is the film thickness, and $A$ is the surface area. The surface energy due to island formation, $E_{\mathrm{sf}}$, is

$$
E_{\text {sf }}=\gamma_{A} A^{\prime},
$$

where $A^{\prime}$ is the area of deformed free surface and $\gamma_{A}$ is the surface tension. From the above equations, it follows that the total energy per unit area $(E / A)$ is

$$
\frac{E}{A}=\frac{E_{\mathrm{st}}}{A}+\frac{E_{\mathrm{sf}}}{A}=\frac{1}{2} G f^{2} t+\gamma_{A} \frac{A^{\prime}}{A}=\frac{1}{2} G\left(\frac{L_{0}-L}{L_{0}}\right)^{2} t+\gamma_{A} \frac{A^{\prime}}{A} .
$$

If $L$ is not consistent with $L_{0}$, islands (or holes) form on the free surface due to stress. In the initial roughening stage, the free surface is deformed as shown in Fig. 2 and then the islands (or holes) grow on the surface. The initial stage of island formation, as in Fig. 2(b), is metastable. If the increase in surface energy is larger than the decrease in strain energy [Fig. 2(b)], islands (or holes) cannot be formed. The increase in surface area in the initial stage of island formation is

$$
A^{\prime}-A=\frac{n\left|L^{\prime}-L\right| A}{\pi\left(L_{0} / 2\right)^{2} L_{0}} \pi L_{0}^{2}=n\left|L^{\prime}-L\right| A \frac{4}{L_{0}},
$$

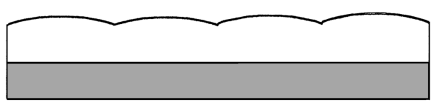

(a)

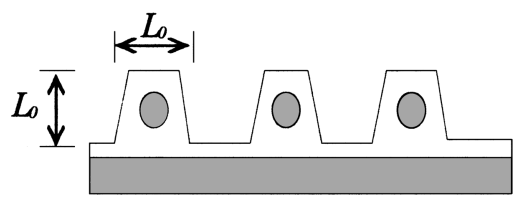

(b)

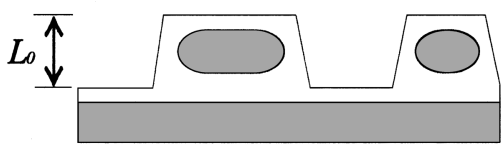

(c)

FIG. 2. Island formation process from the initial roughening stage to the equilibrium state: (a) initial roughening stage, (b) initial stage of island formation, and (c) island growing stage. 
where $L^{\prime}$ is the period adjusted to the initial film thickness $\left(t=n L^{\prime}\right)$. The total energy per unit area $(E / A)$ in the initial stage of island formation is

$$
\frac{E}{A}=\frac{1}{2} G\left(\frac{L_{0}-L}{L_{0}}\right)^{2} n L^{\prime}+\gamma_{A}\left(4 n\left|L^{\prime}-L\right| \frac{1}{L_{0}}+1\right) .
$$

The minimum of the energy with respect to the period $L$ can be obtained by setting the first derivative equal to zero. Because of the term $\left|L^{\prime}-L\right|$, two cases arise, i.e., when $L^{\prime}$ $-L>0$ and when $L^{\prime}-L<0$. For $L^{\prime}>L$, the first derivative is

$$
\frac{\partial(E / A)}{\partial L}=-\frac{n}{L_{0}}\left[G\left(\frac{L_{0}-L}{L_{0}}\right) L^{\prime}+4 \gamma_{A}\right]
$$

and the period of the film corresponding to the minimum energy is

$$
L=L_{0}+\frac{4 \gamma_{A} L_{0}}{G L^{\prime}} \equiv L_{\min },
$$

which follows from Eq. (7), when it is set to zero.

The island topography should result if the corresponding energy is less than the energy corresponding to the flat topography, $(E / A)_{\text {flat }}$, i.e.,

$$
(E / A)_{\text {isle }}<(E / A)_{\text {flat }},
$$

where $(E / A)_{\text {isle }}$ is the minimum energy of Eq. (6) corresponding to $L_{\min }$. The energy $(E / A)_{\text {flat }}$ is simply $(E / A)$ given by Eq. (6) with $L$ replaced by $L^{\prime}$ since the surface would remain flat when $L=L^{\prime}$. But $(E / A)_{\text {isle }}$ is the minimum of Eq. (6) in the interval $L_{0}<L<L^{\prime}$ and therefore Eq. (9) is automatically satisfied. Thus the condition for the island formation is that a minimum with respect to $L$ exists in the interval $L_{0}<L<L^{\prime}$. This condition is

$$
L_{\min }<L^{\prime} .
$$

With the aid of Eq. (8), the condition can be rewritten as follows:

$$
\begin{gathered}
4 \beta<\left(L^{\prime}-L_{0}\right) / L_{0}, \\
\beta \equiv \gamma_{A} /\left(G L^{\prime}\right) .
\end{gathered}
$$

When $L^{\prime}<L$, the period $L$ corresponding to the minimum energy is

$$
L=L_{0}-\frac{4 \gamma_{A} L_{0}}{G L^{\prime}} \equiv L_{\min } .
$$

By the same reasoning as in the case of $L<L^{\prime}$, the criterion for island formation can be written as follows:

$$
4 \beta<\left(L_{0}-L^{\prime}\right) / L_{0} .
$$

Finally, the criteria for the two cases can be combined into

$$
4 \beta<\left|L^{\prime}-L_{0}\right| / L_{0} .
$$

In this light, take as an example the experimental data by Coulon et al. [6] for the use of the criterion. The diblock

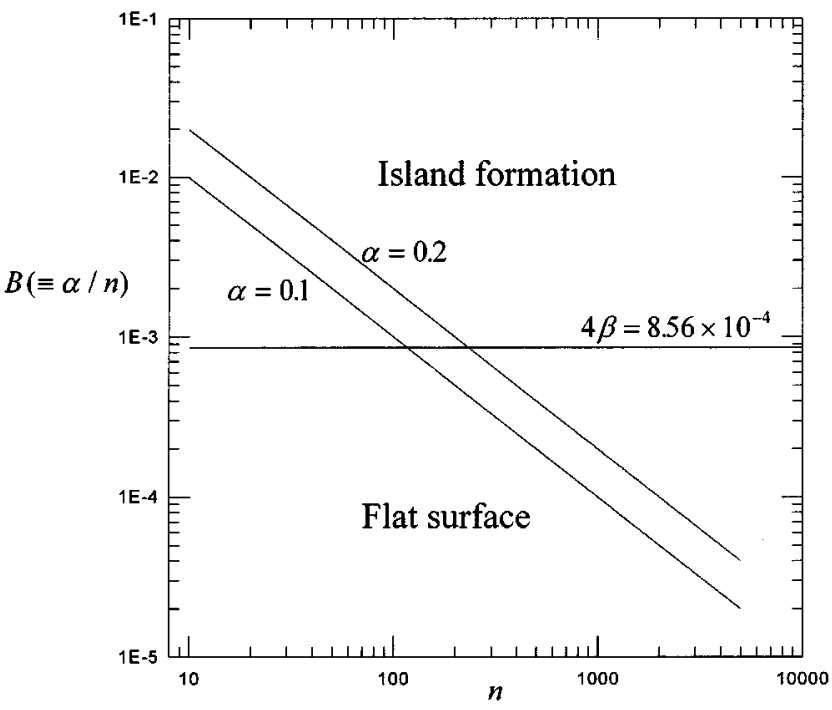

FIG. 3. The morphology of diblock copolymer. The parameters are $\beta=2.14 \times 10^{-4}$ and $0<\alpha<n /(2 n+1)$. When $4 \beta<B$, islands form. When $4 \beta>B$, however, the free surface remains flat.

copolymer they used is polystyrene-(PS) polymethylmethacrylate (PMMA) with an equilibrium period of $39 \mathrm{~nm}$. The initial film thickness was nearly $70 \mathrm{~nm}$ and the morphology that resulted after $47 \mathrm{~h}$ of annealing was island structure. The initial period of the film $L^{\prime}$ was $46.7 \mathrm{~nm}$. The parameter values are $\gamma_{\mathrm{PS}}=33 \mathrm{erg} / \mathrm{cm}^{2}$ and $G=3300 \mathrm{~N} / \mathrm{mm}^{2}$ (PS). Therefore $\beta=2.14 \times 10^{-4}$. Substitution of these values into Eq. (14) shows that the condition is satisfied, which means that the island structure should result as the experiment indicates.

For a given system, one single parameter $\beta$, which is a dimensionless length, determines the morphology. Note in this regard that the critical film thickness at which the morphology goes through a change can also be determined from Eq. (14).

In general, the film thickness $t$ is incompatible with the equilibrium period $L_{0}$, i.e., $t \neq n L_{0}$, where $n$ is either an integer for the symmetric case or an integer plus one-half for the antisymmetric case, such that the period adjusts to $L$. Therefore the number of repeating layers $n$ is related to the film thickness as follows:

$$
t=(n+\alpha) L_{0} .
$$

On the other hand, $t=n L^{\prime}$. Therefore, when $0<\alpha<n /(2 n$ $+1)$, the ratio $\left|L^{\prime}-L_{0}\right| / L_{0}$ becomes $\alpha / n$. When $n /(2 n$ $+1)<\alpha<1$, however, the ratio $\left|L^{\prime}-L_{0}\right| / L_{0}$ becomes (1 $-\alpha) /(n+1)$. Thus the criterion in terms of thickness or $n$ is

$$
\begin{gathered}
4 \beta<B, \\
B \equiv\left\{\begin{array}{l}
\alpha / n \text { for } 0<\alpha<n /(2 n+1) \\
(1-\alpha) /(n+1) \text { for } n /(2 n+1)<\alpha<1 .
\end{array}\right.
\end{gathered}
$$

The critical film thickness is that corresponding to the value of $n$ that satisfies the condition of Eq. (16). This critical thickness or $n$ is shown in Fig. 3 for the example considered 
earlier for two values of $\alpha$. When the film thickness is sufficiently large such that the value of $B$ approaches zero, the condition is violated and the flat surface results.

In summary, a criterion has been derived for determining the morphology of unconfined lamellar diblock copolymers. The critical number of layers leading to the island structure can be determined. The period of the underlying film for the island structure has been derived.
[1] D. G. Walton, G. J. Kellogg, A. M. Mayes, P. Lambooy, and T. P. Russell, Macromolecules 27, 6225 (1994).

[2] G. J. Kellogg, D. G. Walton, A. M. Mayers, P. Lambooy, T. P. Russell, P. D. Gallagher, and S. K. Satija, Phys. Rev. Lett. 76, 2503 (1996).

[3] M. S. Turner, Phys. Rev. Lett. 69, 1788 (1992).

[4] B. Collin, D. Chatenay, G. Coulon, D. Ausserre, and Y. Gallot, Macromolecules 25, 1621 (1992).

[5] M. Maaloum, D. Ausserre, D. Chatenay, G. Coulon, and Y. Gallot, Phys. Rev. Lett. 56, 68 (1992).

[6] G. Coulon, B. Collin, D. Ausserre, D. Chatenay, and T. P.
Russell, J. Phys. (France) 51, 2801 (1990).

[7] N. Koneripalli, N. Singh, R. Levicky, F. S. Bates, P. D. Gallagher, and S. K. Satija, Macromolecules 28, 2897 (1995).

[8] N. Singh, A. Kudrle, M. Sikka, and F. S. Bates, J. Phys. II 5, 377 (1995).

[9] P. Mansky and T. P. Russell, Macromolecules 28, 8092 (1995).

[10] A. M. Mayers, T. P. Russell, P. Bassereau, S. M. Baker, and G. S. Smith, Macromolecules 27, 749 (1994).

[11] J. Coutandin, D. Ehlich, H. Sillescu, and C. H. Wang, Macromolecules 18, 589 (1985). 